\title{
Pushing Boundaries: Women in Sport and Exercise Conference, St Mary's University, Twickenham, June 11-12, 2019
}

\author{
Nicola Brown \\ St Mary's University \\ and Women in Sport and Exercise Academic Network \\ Rachael Bullingham \\ University of Worcester \\ and Women in Sport and Exercise Academic Network
}

\author{
Jacky Forsyth \\ Staffordshire University \\ and Women in Sport and Exercise Academic Network \\ Claire-Marie Roberts \\ University of the West of England \\ and Women in Sport and Exercise Academic Network
}

\section{History of the Conference}

The Women in Sport and Exercise Academic Network (WiSEAN) was established in 2016 to grow, strengthen, and promote research on women in sport and exercise, with the ultimate goal of optimizing women's athletic success and their participation. The impetus for establishing WiSEAN came from the dearth of female-specific research in sport, and in sports medicine in particular (Costello, Bieuzen, \& Bleakley, 2014). This lack of research likely reflects trends where females are often underrepresented in research publications and in other domains, such as science-based subjects (Burton, 2015; Ceci \& Williams, 2011; Krishnamurthy, Liao, Deveaux, \& Dalecki, 2017; Pape, 2019; Shor, van de Rijt, Miltsov, Kulkarni, \& Skiena, 2015). It was felt that organizing a network, where individuals could collaborate, mentor, support, and disseminate their work on the female sports person or participant, could go some way in addressing these sex and gender inequalities.

An inaugural meeting of WiSEAN was held on September 14, 2016, with one of the agreed outcomes being to hold a regular conference for the purpose of disseminating cutting-edge research associated with all aspects of the exercising female. The first annual Women in Sport and Exercise (WISE) conference, Blood, Sweat, and Fears, was held in the United Kingdom at Staffordshire University in 2018 (Roberts \& Forsyth, 2019). In addition to the annual conference, the network enables academics and researchers to communicate and collaborate with each other, to provide a platform for promoting research, and to support members via a mentoring scheme for those who want to develop their research skills in a relevant field. Membership of WiSEAN is open to all individuals over the age of 18 who support the WiSEAN aims. As of the end of 2019, the network has 276 members internationally. Further details of WiSEAN's work can be found on the website: https://www.wisean.net/.

\footnotetext{
The authors are with the Women in Sport and Exercise Academic Network, United Kingdom. Brown is with St Mary's University, Twickenham, United Kingdom. Forsyth is with Staffordshire University, Stoke-on-Trent, United Kingdom. Bullingham is with the University of Worcester, Worcester, United Kingdom. Roberts is with the University of the West of England, Bristol, United Kingdom. Brown (nicola.brown@stmarys.ac.uk) is corresponding author.
}

\section{Introduction to the 2019 Conference}

Opportunities for women in sport and exercise are expanding rapidly, whether it is leading a governing body, excelling in elite competition, or simply participation for fitness, camaraderie, and the love of sport. However, there is still much work to do to achieve a level playing field. The 2019 conference program was based around the theme of Pushing Boundaries and aimed to bring together leading thinkers and practitioners from a range of disciplines to promote discussion and debate around the issues that females face in sport and exercise at all levels. In addition, it focused on how the industry can innovate, challenge attitudes, and push the boundaries that still exist for women in sport.

The conference explored the latest research and real-world evidence to address female-specific issues and life stages that impact on sport or exercise performance and health. It explored sex and gender inequality in the sport and exercise arena and provided occasion to discuss the opportunities and issues surrounding female participation in physical activity. Key areas of focus were the practical application of research to increase the opportunities for females in sport and exercise and minimize and overcome identified barriers.

The Conference Committee comprised of Dr. Nicola Brown (Chair, St Mary's University), Dr. Jacky Forsyth (Staffordshire University), Dr. Claire-Marie Roberts (UWE Bristol), Dr. Rachael Bullingham (University of Worcester), and Dr. Charles Pedlar (St Mary's University). Collectively their expertise spans a number of disciplines in women's health including anatomy, physiology, psychology, sports sociology, and culture.

\section{Summary of Keynotes}

The 2019 WISE Conference opened with an inspiring keynote session from Professor Greg Whyte, OBE, and four-time Great Britain (GB) Winter Olympian Chemmy Alcott. This unique pairing provided a platform to debunk, explain, and answer questions regarding exercise during pregnancy with Professor Whyte offering theoretical insights, and Chemmy Alcott sharing her personal experiences of exercise before, during, and after pregnancy.

Further consideration of female life-stages continued with presentations from Dr. Claire-Marie Roberts and Professor Mark De Ste Croix, with their presentations on insights into motherhood 
and the elite female athlete, and developing robustness and readiness in female youth athletes, respectively. Dr. Roberts provided an overview of the myriad social and psychological barriers that female elite athlete mothers face when returning to sport, from balancing the dual roles of elite athlete and motherhood, to the withdrawal of funding and sponsorship support during pregnancy. Professor De Ste Croix highlighted the importance of applying sexspecific and maturation-specific models to the female youth athlete to enhance performance and reduce injury.

Having worked in anti-doping for over 20 years, Nicole Sapstead shared her experience of top-flight sports governance detailing her journey to becoming CEO of UK Anti-Doping. Providing insight to the obstacles and challenges she faced along the way as a woman generally, and, more specifically, as one who works within the male dominated area of sport, her inspiring talk left the audience convinced that "there is no such word as can't."

Having represented GB more times than any other female hockey player in history, Kate-Richardson Walsh focused on how to empower individual growth for team success and how the GB team built the incredible team ethos and commitment that drove them to a nail-biting victory at the Rio Olympic Games. Kate shared the team's process of self-examination and rebuilding that occurred after a disastrous low of 11th place at the World Cup in 2014, in which the team developed and signed up to a collective culture or behavior, created a sense of common purpose, and installed distributed leadership to ensure it was "lived and breathed" every day.

We also heard from Dr. Richard Burden who detailed how the English Institute of Sport (EIS) have been evaluating the best ways to support, inform, and empower elite female athletes and those who support them. Providing an overview of SmartHER, a program launched by the EIS with the objective of enabling elite female athletes to thrive and fulfil their performance potential in a system optimized to support individual differences, Dr. Burden focused on two key areas: (1) understanding and optimizing menstrual health in elite female athletes; and (2) understanding how hormonal fluctuations physiologically and psychologically influence preparedness for and responses to training and performance.

Linked to female-specific health issues, Professor Joanna Wakefield-Scurr drew attention to the issues exercising females can experience as a result of breast bounce, including breast pain, discomfort, and embarrassment. With one in four adult women, and half of adolescent girls reporting the breast as a barrier to physical activity, Professor Wakefield-Scurr discussed the benefits of appropriate breast support during sports and exercise and the importance of educating recreationally active women, elite sports women, and their support teams on this important aspect of women's health.

Turning to sports participation and engagement, Tanya Martin form Women in Sport put the spotlight on teenage girls. She highlighted that research has been repeatedly shown that girls are less physically active than boys and that this worsens with age. As a consequence, Women in Sport have been focused on how to reframe sport and physical activity to have both relevance and appeal for girls. The presentation reviewed the personal, social, and contextual barriers to sport that girls face and shared the work Women in Sport have been doing to address the challenge of girls disengaging with sport.

\section{Conference Review}

For a complete list of 2019 WISE conference abstracts, please see this issue of the Women in Sports and Physical Activity Journal. The conference attracted over 100 international delegates from a variety of backgrounds and disciplines, all united in their common interest in women in sport and exercise. The evaluation of the conference feedback showed an overwhelmingly positive response to the event with many commenting that the keynotes were among the best they had heard, and that the variety of speakers exposed them to challenges that women in sport face that they had not previously considered. Multiple delegates commented that they found Kate RichardsonWalsh's keynote speech particularly inspiring, and that the inclusion of speakers with 'real-life' experience was a particular strength of the conference program. Some other example statements received from attendees are below:

- "My colleagues and I learnt so much from the fantastic variety of speakers across the two days."

- "The WiSEAN conference had a great range of interesting speakers from a variety of backgrounds."

- "The organization of sessions was great, and the breakout sessions were very interesting."

- "It was great to share good practice and develop collaborations for the future."

\section{Looking Ahead}

The third WISE conference will take place at the University of Worcester on November 11-12, 2020. This conference will maintain the multidisciplinary approach of previous years with the focus on Redressing the Balance for women's sport. The purpose is to highlight research and raise awareness to address inequalities that women face in sport. Additionally, this conference will seek to share good practice and improve the impact of research and initiatives. Uniquely, this event will be running in a parallel conference for a European Commission funded Erasmus+ project, which the University of Worcester is leading with six other international partners, entitled the Gender Equity Toolkit for Generation Z.

\section{References}

Burton, L.J. (2015). Underrepresentation of women in sport leadership: A review of research. Sport Management Review, 18(2), 155-165. doi:10.1016/j.smr.2014.02.004

Ceci, S.J., \& Williams, W.M. (2011). Understanding current causes of women's underrepresentation in science. Proceedings of the National Academy of Sciences, 108(8), 3157-3162. doi:10.1073/pnas.1014871108

Costello, J.T., Bieuzen, F., \& Bleakley, C.M. (2014). Where are all the female participants in Sports and Exercise Medicine research? European Journal of Sport Science, 14(8), 847-851. PubMed ID: 24766579 doi:10.1080/17461391.2014.911354

Krishnamurthy, M., Liao, S., Deveaux, M., \& Dalecki, M. (2017). The underrepresentation of women in prestigious ethics journals. Hypatia, 32(4), 928-939. doi:10.1111/hypa.12351

Pape, M. (2019). Gender segregation and trajectories of organizational change: The underrepresentation of women in sports leadership. Gender \& Society, 34(1), 81-105. doi:10.1177/0891243219867914

Roberts, C.-M., \& Forsyth, J. (2019). The inaugural Women in Sport and Exercise Conference: Consensus Statement. Women in Sport and Physical Activity Journal, 27(1), 60-62. doi:10.1123/wspaj.2019-0004

Shor, E., van de Rijt, A., Miltsov, A., Kulkarni, V., \& Skiena, S. (2015). A paper ceiling. American Sociological Review, 80(5), 960-984. doi:10. $1177 / 0003122415596999$ 